\title{
Suppression of Runaway Electrons by Resonant Magnetic Perturbations in TEXTOR Disruptions
}

\author{
M. Lehnen, S. A. Bozhenkov, S. S. Abdullaev, and TEXTOR Team \\ Institute of Energy Research-Plasma Physics, Forschungszentrum Jülich GmbH, EURATOM Association, \\ Trilateral Euregio Cluster, D-52425 Jülich, Germany \\ M. W. Jakubowski \\ Max-Planck-Institut für Plasmaphysik, IPP-EURATOM Association, Teilinstitut Greifswald, \\ Wendelsteinstrasse 1, 17491 Greifswald, Germany \\ (Received 24 April 2008; published 24 June 2008)
}

\begin{abstract}
The generation of runaway electrons in the international fusion experiment ITER disruptions can lead to severe damage at plasma facing components. Massive gas injection might inhibit the generation process, but the amount of gas needed can affect, e.g., vacuum systems. Alternatively, magnetic perturbations can suppress runaway generation by increasing the loss rate. In TEXTOR disruptions runaway losses were enhanced by the application of resonant magnetic perturbations with toroidal mode number $n=1$ and $n=2$. The disruptions are initiated by fast injection of about $3 \times 10^{21}$ argon atoms, which leads to a reliable generation of runaway electrons. At sufficiently high perturbation levels a reduction of the runaway current, a shortening of the current plateau, and the suppression of high energetic runaways are observed. These findings indicate the suppression of the runaway avalanche during disruptions.
\end{abstract}

DOI: 10.1103/PhysRevLett.100.255003

PACS numbers: 52.55.Fa, 52.25.Xz, 52.35.Vd

Introduction. - Runaway currents of several megaampere (MA) are expected to be generated in ITER disruptions by avalanche multiplication [1]. An uncontrolled loss of this high energetic electron beam to the plasma facing components might lead to serious damages. The avoidance of runaways is therefore mandatory. Presently, massive gas injection is discussed as a technique to mitigate forces and heat loads and to suppress runaway generation. However, it might appear that the latter aim requires enormous amounts of gas to be injected into the vacuum vessel. In order to overcome the critical electric field for the runaway avalanche process in ITER, the injection of up to $5 \times 10^{25}$ atoms might be necessary, taking into account the poor mixing efficiencies of the order of 10\%-30\% and the estimated electric field of $40 \mathrm{~V} / \mathrm{m}$ for ITER [1]. Such a huge amount of gas might have significant implications on vacuum systems. Moreover, in present-day devices, these required densities for avalanche suppression have not been reached yet.

Magnetic perturbations can be an alternative mechanism for runaway suppression in disruptions [2]. However, until now experiments with externally excited perturbation fields have been conducted only in JT-60 [3]. These experiments have shown that runaways are absent for a sufficient high perturbation field with base mode number of $m / n=$ $3 / 2$. The effect of resonant magnetic perturbations (RMP) on runaway generation has also been studied at TEXTOR during the flattop phase of low density discharges $[4,5]$. The perturbation field is produced by the coils of the dynamic ergodic divertor (DED) [6]. Application of a $m / n=6 / 2$ perturbation field caused a significant decrease of the runaway population above a certain threshold. Enhanced losses were also seen in Tore Supra applying the ergodic divertor during flattop [7]. In this Letter we present a systematic study of runaway suppression with RMP $(n=1,2)$ during disruptions. We show that the avalanche multiplication of runaways can be suppressed by RMP.

Experimental setup.-Runaway electrons are deliberately generated by injection of large amounts of argon by a fast disruption mitigation valve (DMV) [8]. The argon cools the edge plasma up to the $q=2$ surface and initiates then the thermal quench. However, pure argon injection has a low mixing efficiency and the rise in electron density by the gas injection is moderate. This causes the ratio of Dreicer field to the induced electrical field to be low

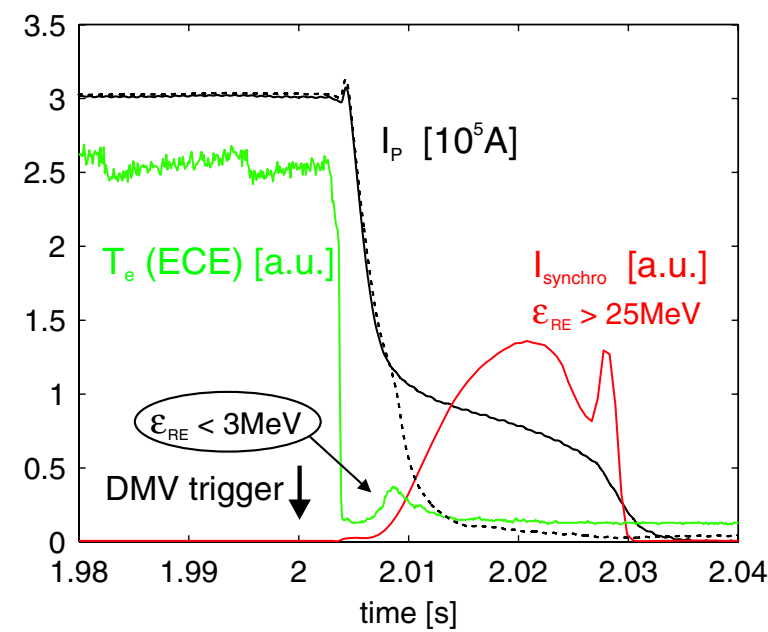

FIG. 1 (color online). Time traces of the plasma current $I_{P}$, the electron temperature $T_{e}$ measured by ECE, and the synchrotron radiation $I_{\text {synchro }}$ measured by the infrared camera. The dashed line gives the current decay of a pulse with maximum perturbation amplitude in $n=2$. 
enough (about 40-50) to initiate generation of runaways. In this way, runaway electrons can be reliably generated in TEXTOR disruptions. Further details can be found in $[9,10]$. A typical evolution of such a disruption is given in Fig. 1. The parameters of these Ohmic discharges are plasma current $I_{P}=300 \mathrm{kA}$, toroidal magnetic field $B_{t}=$ $2.25 \mathrm{~T}$, line averaged central density $n_{e}=2.0 \times 10^{19} \mathrm{~m}^{-3}$, major radius $R=1.75 \mathrm{~m}$, and minor radius $a=0.46 \mathrm{~m}$. The disruption valve is triggered at $t=2.0 \mathrm{~s}$ and about $3 \times$ $10^{21}$ argon atoms are injected into the TEXTOR vessel. After 3-4 ms the thermal quench occurs. During the following current quench, a runaway current plateau forms, which can last up to $50 \mathrm{~ms}$. Runaway generation is also seen on the synchrotron radiation measured for energies below about $3 \mathrm{MeV}$ by the electron cyclotron emission (ECE) diagnostic [5] and for high energies above about $25 \mathrm{MeV}$ by the infrared camera viewing the plasma tangentially [11].

Resonant magnetic perturbations can be applied at TEXTOR by the DED [6]. This divertor consists of 16 helical coils mounted at the high field side of TEXTOR. The base mode of the perturbation spectrum can be chosen by different current distributions in the coils to be $m / n=$ $12 / 4,6 / 2$, or $3 / 1$. For the runaway studies presented here, the DED was energized about $200 \mathrm{~ms}$ before the valve was triggered. Because of the extensive hardware work necessary for mode changes, only toroidal mode numbers $n=1$ and 2 have been investigated during the experiments reported here.
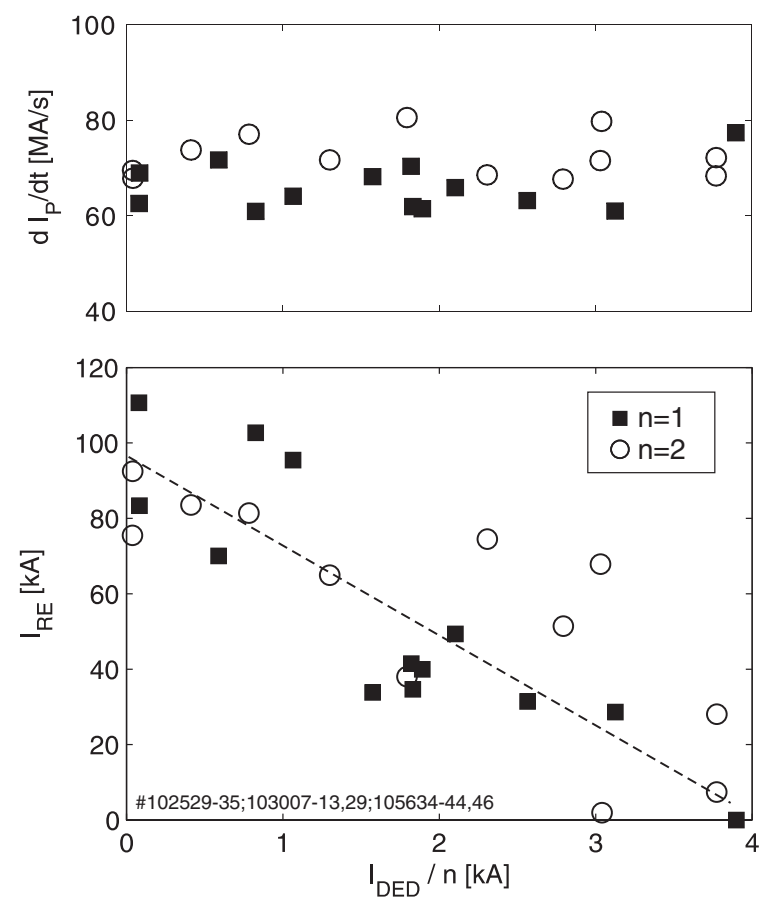

FIG. 2. Maximum runaway current as function of the coil current divided by the toroidal mode number of the perturbation field. The open symbols represent the maximum current decay rate.
Runaway suppression. - The dashed line in Fig. 1 gives an example for the current evolution with full $n=2$ perturbation amplitude. The runaway electrons are almost completely suppressed, only a small plateau is visible. The runaway current was estimated by subtracting the current evolution of a disruption with fully suppressed runaway generation, i.e., with exponential current decay. Figure 2 shows the maximum runaway current as a function of the coil current $I_{\mathrm{DED}}$ divided by the toroidal mode number $n$, which is proportional to the amplitude of the radial field at the coil position. A clear reduction of the runaway current with increasing perturbation is observed. However, the data show a large scatter and a 100\% suppression probability cannot be achieved, even for maximum perturbation. Possible reasons are discussed below. The maximum current decay rate, also given in Fig. 2, is not influenced by the perturbation field. The values lie between 60 and $80 \mathrm{MA} / \mathrm{s}$. We therefore conclude that the electric field and the impurity content are constant during these experiments. The plasma current is measured outside the vacuum vessel and therefore also includes the current induced in the vessel structure. Thus, the measured decay rate can be lower than the plasma current decay rate.

At high amplitudes tearing modes are excited by the DED with mode number $m / n=3 / 1$ and $m / n=3 / 2$ before the disruption. Because of the current redistribution during the energy quench, these tearing modes are assumed to be of no relevance in the current decay phase. This has also been tested by exciting the tearing mode in $n=1$ configuration at $I_{\mathrm{DED}}=2.5 \mathrm{kA}$ and a subsequent reduction of the DED current to $I_{\mathrm{DED}}=0.5 \mathrm{kA}$ before the disruption. This causes the tearing mode to stay locked to the perturbation field. In this disruption, no sign of runaway suppression was observed.

The reduction of the runaway current is accompanied by a shortening of the runaway current plateau. The length of the plateau is given in Fig. 3 as the time the plasma current

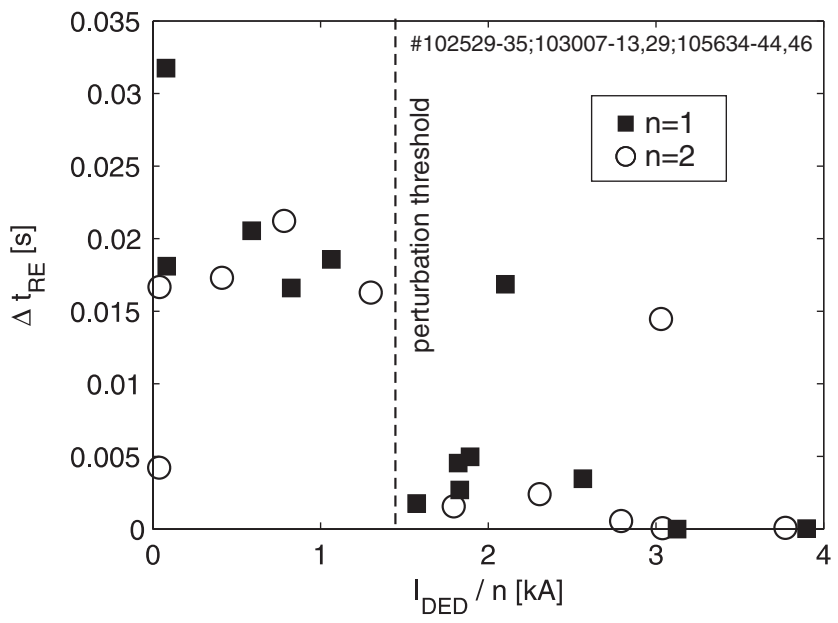

FIG. 3. Length of the runaway plateau. $\Delta t_{\mathrm{RE}}$ is the time between $I_{P}=150 \mathrm{kA}$ and $I_{P}=30 \mathrm{kA}$, where the offset from pure exponential decay was subtracted. 
needs to drop from $I_{P}=150$ to $30 \mathrm{kA}$. The time for the exponential decay without runaway plateau is subtracted. Beside a few outliers, a clear threshold can be identified at about $I_{\mathrm{DED}} / n=1.4 \mathrm{kA}$, beyond which the runaway plateau is significantly shorter. For $n=1$ perturbation, such a threshold can also been seen in the maximum runaway current (Fig. 2). Above the threshold the runaway current is reduced by a factor of $2-3$. The scatter of the $n=2$ data does not allow the identification of a threshold. The scatter might be related to the reduced penetration depth of the perturbation field for $n=2$ : the radial decay of the field is proportional to $r^{m-1}$. The positioning of the runaway can thus be more essential for suppression than it is for $n=1$. The influence of the beam position will be discussed below. It should be mentioned here that field screening as it is discussed for the divertor topology or for edge localized mode mitigation experiments with RMP is not to be expected, because of the low temperatures during the current decay phase $(5-25 \mathrm{eV})$ and low rotation velocities.

The intensity of the synchrotron radiation was measured from $R=1.4$ to $1.9 \mathrm{~m}$ and the maximum of the radial integral is given in Fig. 4. Again a significant drop to almost zero synchrotron radiation can be observed at $I_{\mathrm{DED}} / n=1.4 \mathrm{kA}$. Thus, for perturbation fields above this threshold, runaways are lost before they reach energies above $25 \mathrm{MeV}$. The avalanche rate becomes relevant if the runaways can achieve energies above about 10$20 \mathrm{MeV}$ [12]. The absence of these high energetic electrons indicates the suppression of the avalanche.

Discussion. - With RMP it is only possible to suppress avalanche generation of runaways. The generation rate for the Dreicer mechanism increases exponentially with electric field and cannot be compensated by RMP induced losses. The enhanced diffusion of thermal particles during RMP is related to a radial field line diffusion, which is described by a field line diffusion coefficient $D_{\mathrm{FL}}$. High energetic electrons, however, are displaced with respect to

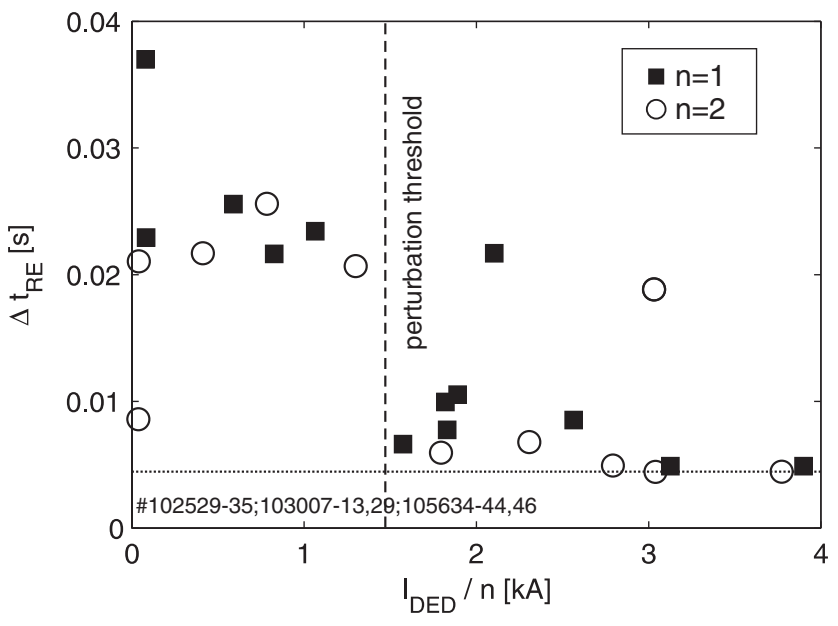

FIG. 4. Maximum of the radial integral of the synchrotron radiation $I_{\text {synchro }}$ during the current decay as function of the perturbation amplitude. the magnetic field line structures. It is therefore necessary to calculate the guiding-center motion of the runaways to quantify the degree of perturbation. This has been done using the relativistic formulation of Hamiltonian equations for the guiding-center motion, which was integrated by the mapping method [13]. The field line diffusion is then replaced by a particle trajectory diffusion $D_{\mathrm{PT}}$.

The critical diffusion coefficient $D_{\mathrm{PT}}$ for the particle trajectories needed for suppression is given in Fig. 5. The dotted line is the minimum diffusion coefficient needed to compensate the primary generation rate (Dreicer). The solid line is the minimum diffusion coefficient to compensate the avalanche rate. They are estimated from the balance $D_{\perp} \partial^{2} n_{\mathrm{RE}} / \partial r^{2}=G_{\mathrm{RE}}$, with $G_{\mathrm{RE}}$ being the runaway generation rate (Dreicer or avalanche). The diffusion coefficient follows from $D_{\mathrm{PT}}=D_{\perp} / c$, taking into account that the runaways move at the speed of light. The runaway generation rate is calculated according to the standard equations, which are given, for example, in $[10,14]$. The generation of primary runaways is not influenced by field diffusion and depends only on the electron density and temperature. The critical $D_{\mathrm{PT}}$ for the avalanche suppression has a relatively weak dependence on the electric field and has values achievable with external perturbation coils.

Figure 6 shows examples of the structure seen by electrons with an energy of $5 \mathrm{MeV}$. For these calculations it is assumed that a total current of $80 \mathrm{kA}$ is carried by runaway electrons. The radius of the runaway beam is $0.2 \mathrm{~m}$, as indicated by soft x-ray measurements, which will be reported elsewhere. Three cases are shown, with different horizontal position of the runaway beam. Almost complete ergodization is found for a position close to the perturbation coils $(R=1.55 \mathrm{~m})$. In this case, the beam is about $8 \mathrm{~cm}$ away from the DED plates. For the center position $(R=1.75 \mathrm{~m})$, the beam has - beside small island struc-

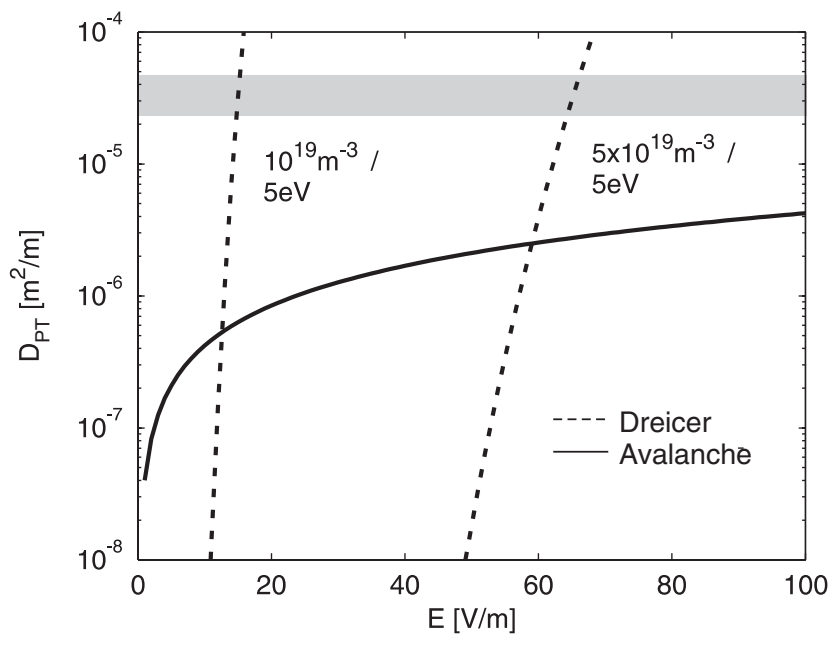

FIG. 5. Critical field line diffusion coefficient for runaway suppression as function of the parallel electric field. The gray region indicates the diffusion coefficient which can be achieved for $n=1$ perturbations [case (a) in Fig. 6]. 


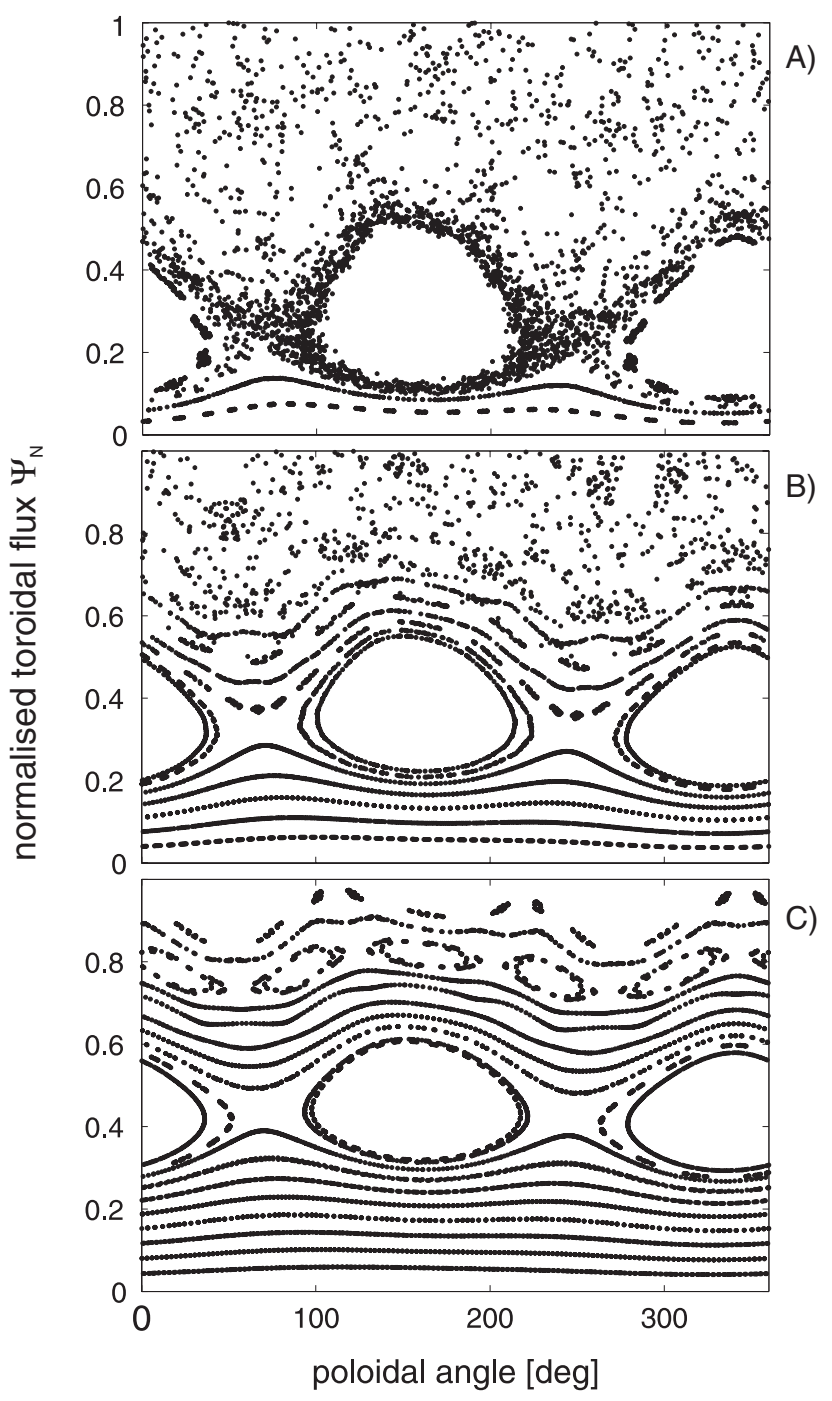

FIG. 6. Poincaré plots for different radial positions of the runaway beam: (a) $R=1.55 \mathrm{~m}$, (b) $R=1.65 \mathrm{~m}$, (c) $R=1.75 \mathrm{~m}$. The radius of the runaway beam is $r=0.2 \mathrm{~m}$. The DED is in $n=1$ configuration at the maximum amplitude of $3.75 \mathrm{kA}$ per coil.

tures - intact flux surfaces. This example shows the importance of the position of the runaway beam with respect to the perturbation coils: in case (a) a perturbation enhanced loss is expected over the full radial extent of the runaway beam, whereas in case (c) runaway diffusion will not be enhanced. Another important parameter is the runaway energy: the higher the energy, the more the orbits are shifted away from the coils towards the low field side. Moreover, without a detailed knowledge about the radial current distribution, such calculations can only be examples. Here, we assumed $j \sim\left(1-\rho^{2}\right)^{\nu}$, with peaking $\nu=1.5$. A detailed parameter variation is beyond the scope of this Letter. The effective diffusion coefficient for the particle orbits for case $A$ in Fig. 6 varies between $2.8 \times 10^{-5} \mathrm{~m}^{2} / \mathrm{m} \quad\left(\Psi_{N}=0.4\right)$ and $4.5 \times 10^{-5} \mathrm{~m}^{2} / \mathrm{m}$ $\left(\Psi_{N}=1.0\right)$. This range is well above the requirement for avalanche suppression as indicated in Fig. 5. The corresponding effective particle diffusion coefficient is of the order $10^{4} \mathrm{~m}^{2} / \mathrm{s}$, assuming that the electrons have the speed of light.

The runaway beam position could also explain the two outliers in Figs. 3 and 4, where runaways were not suppressed at high DED amplitudes. The birth position of the runaways might be determined by parameters which are not well controlled in disruptions. In the case where the runaways are generated farther away from the DED coils, the perturbation is insufficient and a runaway beam builds up. In this respect, the vertical field can play a significant role. It will be shown elsewhere that a clear correlation between the movements of the runaway beam and the applied vertical field exists. However, a correlation between DED amplitude and vertical field, which would give a trivial explanation for the early loss of the runaway beam, is not found.

Conclusions. - The generation of runaway electrons was suppressed in TEXTOR disruptions by applying perturbation fields with $n=1$ and $n=2$. A significant reduction of the runaway current was seen. However, a reliable complete suppression could not be achieved until now. The runaway plateau was shortened significantly above a perturbation threshold of about $1.4 \mathrm{kA} / n$. The generation of runaways with energies above $25 \mathrm{MeV}$ was suppressed. All this indicates that a successful suppression of the avalanche multiplication was achieved. The influence of the runaway beam position has to be investigated further. It might be necessary to either optimize the coil position in future experiments or to establish a reliable control mechanism for the runaway position.

[1] T. C. Hender et al., Nucl. Fusion 47, S128 (2007).

[2] P. Helander, L.-G Eriksson, and F. Andersson, Phys. Plasmas 7, 4106 (2000).

[3] R. Yoshino and S. Tokuda, Nucl. Fusion 40, 1293 (2000).

[4] K. H. Finken et al., Nucl. Fusion 46, S139 (2006).

[5] K. H. Finken et al., Nucl. Fusion 47, 91 (2007).

[6] K.H. Finken et al., Energy Technology (Forschungszentrum Jülich GmbH, Jülich, Germany, 2005), Vol 45.

[7] Ph. Ghendrih et al., Plasma Phys. Controlled Fusion 38, 1653 (1996).

[8] S. A. Bozhenkov et al., Rev. Sci. Instrum. 78, 033503 (2007).

[9] S. A. Bozhenkov et al., in Proceedings of the 34th European Physical Society Conference on Plasma Physics, Warsaw, 2007 (European Physical Society, Mulhouse, France, 2007), Vol. 31F, p. 5.123.

[10] S. A. Bozhenkov et al., Plasma Phys. Controlled Fusion (to be published).

[11] K. H. Finken et al., Nucl. Fusion 30, 859 (1990).

[12] R. Jayakumar, H. H. Fleischmann, and S. J. Zweben, Phys. Lett. A 172, 447 (1993).

[13] A. Wingen et al., Nucl. Fusion 46, 941 (2006).

[14] P. Helander, L.-G. Eriksson, and F. Andersson, Plasma Phys. Controlled Fusion 44, B247 (2002). 